\title{
A BAXTER TYPE ESTIMATOR OF AN UNKNOWN PARAMETER OF THE COVARIANCE FUNCTION IN THE NON-GAUSSIAN CASE
}

UDC 519.21

\author{
O. O. SYNYAVS'KA
}

\begin{abstract}
The problem of estimation of a parameter of the covariance function is studied for a non-Gaussian stochastic process. Non-asymptotic confidence intervals for the estimator are constructed by using the Baxter statistics.
\end{abstract}

\section{INTRODUCTION}

Limit theorems for the mean square convergence or for the almost sure convergence of Baxter sums to a positive constant are called the Lévy-Baxter theorems or Baxter type theorems. These limit theorems for Baxter sums constructed from stochastic processes and random fields were considered by P. Lévy 16, G. Baxter [10, E. G. Gladyshev 3, S. M. Berman [11, T. Kawada [14, S. M. Krasnitskiü [5], and others.

E. P. Besklins'ka and Yu. V. Kozachenko 1] proved several Baxter type theorems for pre-Gaussian stochastic processes. Lévy-Baxter theorems for jointly strictly subGaussian and jointly pseudo-Gaussian stochastic processes were studied in the monograph by V. V. Buldygin and Yu. V. Kozachenko [2]. O. O. Kurchenko [7 proved a Baxter type theorem for jointly sub-Gaussian random fields. Yu. V. Kozachenko and O. O. Kurchenko [15] obtained some Lévy-Baxter theorems for a certain class of nonGaussian stochastic processes.

Various Baxter type theorems for stochastic processes and random fields were used by R. E. Maüboroda [8], Yu. V. Kozachenko and O. O. Kurchenko [4], O. O. Kurchenko 6], J.-C. Breton, I. Nourdin, and G. Peccati [12] and others for the estimation of parameters of covariance functions.

\section{Setting of the PROBlem}

Let $(\Omega, F, \mathrm{P})$ be a probability space.

Definition 2.1 ([15]). We say that a random vector $(\xi, \eta) \in L_{4}(\Omega) \times L_{4}(\Omega)$ possesses property $K$ if

(1) $\mathrm{E} \xi=\mathrm{E} \eta=0$,

(2) $\mathrm{E}(\xi \pm \eta)^{4} \leq 3\left(\mathrm{E}(\xi \pm \eta)^{2}\right)^{2}$.

The subclass $K_{1}$ is defined as the set of all vectors of the class $K$ for which

$$
\mathrm{E}(\xi \pm \eta)^{4}=3\left(\mathrm{E}(\xi \pm \eta)^{2}\right)^{2}
$$

2010 Mathematics Subject Classification. Primary 42C40; Secondary 60G12.

Key words and phrases. Baxter sums, confidence interval, stochastic processes with increments belonging to the class $K$. 
Definition $2.2([15])$. A centered stochastic process $\{X(t), t \in[0,1]\}$ is called a process with the first and second order increments of the class $K$ if the random vectors $\left(\xi_{1}, \eta_{1}\right)$ and $\left(\xi_{2}, \eta_{2}\right)$ possess property $K$ for all $0 \leq s \leq t \leq u \leq v \leq 1$, where

$$
\begin{aligned}
\xi_{1}=X(t)-X(s), \quad \eta_{1} & =X(v)-X(u), \quad \xi_{2}=X(t)-2 X\left(\frac{t+s}{2}\right)+X(s), \\
& \eta_{2}=X(v)-2 X\left(\frac{u+v}{2}\right)+X(u) .
\end{aligned}
$$

A stochastic process $\{X(t), t \in[0,1]\}$ is called a process with the first and second order increments of the class $K_{1}$ if the random vectors $\left(\xi_{1}, \eta_{1}\right)$ and $\left(\xi_{2}, \eta_{2}\right)$ defined as above belong to the class $K_{1}$.

For example, centered Gaussian stochastic processes have increments of the first and second order of the class $K_{1}$.

Note that if increments of the first and second order of a stochastic process

$$
\{\xi(t), t \in[0,1]\}
$$

belong to the class $K_{1}$, then the increments $\xi_{i}, i=1,2$, are such that

$$
\mathrm{E} \xi_{i}^{4}=3\left(\mathrm{E} \xi_{i}^{2}\right)^{2}, \quad i=1,2 .
$$

Let $\{\xi(t), t \in[0,1]\}$ be a centered stochastic process with the covariance function depending on a parameter $H \in(0,1)$,

$$
r(t, s)=\frac{1}{2}\left(|t|^{2 H}+|s|^{2 H}-|t-s|^{2 H}\right), \quad t, s \in[0,1],
$$

and let its increments of the first and second order belong to the class $K_{1}$. We want to construct an estimator of the unknown parameter $H$ and a non-asymptotic confidence interval by using the observations of a stochastic process $\{\xi(t), t \in[0,1]\}$ at points

$$
\left\{\frac{k}{a_{n}}, \frac{k+0.5}{a_{n}} \mid 0 \leq k \leq a_{n}, n \geq 1\right\},
$$

where the sequence $\left(a_{n}\right) \subset \mathbf{N}$ is such that $a_{n} \rightarrow+\infty$ as $n \rightarrow \infty$.

In what follows we assume that the sequence $\left(a_{n}\right)$ has the property that the series $\sum_{n=1}^{\infty} a_{n}^{-\alpha}$ converges for all numbers $\alpha>0$.

The fractional Brownian motion is an example of a stochastic process with covariance function (11) and with increments of the first and second order of the class $K_{1}$. Below we provide an example of a non-Gaussian stochastic process with covariance function (1) and with increments of the first and second order of the class $K_{1}$.

Example 2.1. It is shown in [13, that the fractional Brownian motion $\left\{B_{H}(t), t \in[0,1]\right\}$ with the Hurst parameter $H \in\left(\frac{1}{2}, 1\right)$ admits the following representation

$$
B_{H}(t)=\sum_{n=1}^{\infty} \frac{\sin \left(x_{n} t\right)}{x_{n}} X_{n}+\sum_{n=1}^{\infty} \frac{1-\cos \left(y_{n} t\right)}{y_{n}} Y_{n}, \quad t \in[0,1]
$$

where $\left(X_{n}\right)$ and $\left(Y_{n}\right)$ are two independent sequences, each consisting of independent Gaussian random variables such that $\mathrm{E} X_{n}=\mathrm{E} Y_{n}=0, n \geq 1$, and

$$
\begin{aligned}
\operatorname{Var} X_{n} & =2 c_{H}^{2} x_{n}^{-2 H} J_{1-H}^{-2}\left(x_{n}\right), \\
\operatorname{Var} Y_{n} & =2 c_{H}^{2} y_{n}^{-2 H} J_{-H}^{-2}\left(y_{n}\right) ;
\end{aligned}
$$

$c_{H}^{2}=\pi^{-1} \Gamma(1+2 H) \sin \pi H ; J_{\nu}, \nu \neq-1,-2, \ldots$, is the Bessel function of the first kind and of order $\nu ;\left(x_{n}\right)$ is the sequence of positive zeros of the Bessel function $J_{-H}$ written in ascending order; $\left(y_{n}\right)$ is the sequence of positive zeros of the Bessel function $J_{1-H}$ written in ascending order. 
Further let $\left(\xi_{n}\right)$ and $\left(\eta_{n}\right)$ be two independent sequences each consisting of independent random variables such that

$$
\begin{gathered}
\mathrm{E} \xi_{n}=\mathrm{E} \eta_{n}=0, \quad \mathrm{E} \xi_{n}^{4}=3\left(\mathrm{E} \xi_{n}^{2}\right)^{2}, \quad \mathrm{E} \eta_{n}^{4}=3\left(\mathrm{E} \eta_{n}^{2}\right)^{2} \\
\operatorname{Var} \xi_{n}=\operatorname{Var} X_{n}, \quad \operatorname{Var} \eta_{n}=\operatorname{Var} Y_{n} .
\end{gathered}
$$

Then the stochastic process

$$
\xi(t)=\sum_{n=1}^{\infty} \frac{\sin \left(x_{n} t\right)}{x_{n}} \xi_{n}+\sum_{n=1}^{\infty} \frac{1-\cos \left(y_{n} t\right)}{y_{n}} \eta_{n}, \quad t \in[0,1]
$$

has covariance function (11) and its increments of the first and second order belong to the class $K_{1}$. Now we prove that the first series on the right-hand side of (44) converges in $L_{4}(\Omega)$ for an arbitrary $t \in[0,1]$. The proof of the convergence of the second series is the same. Let $1 \leq N<M$. Applying the Hölder inequality with $p=\frac{4}{3}$ and $q=4$, we get

$$
\left|\sum_{n=N}^{M} \frac{\sin x_{n} t}{x_{n}} \xi_{n}\right| \leq \sum_{n=N}^{M} \frac{1}{x_{n}^{1-\lambda}} \frac{\left|\xi_{n}\right|}{x_{n}^{\lambda}} \leq\left(\sum_{n=N}^{M} \frac{1}{x_{n}^{4(1-\lambda) / 3}}\right)^{3 / 4}\left(\sum_{n=N}^{M} \frac{\xi_{n}^{4}}{x_{n}^{4 \lambda}}\right)^{1 / 4}
$$

for all $\lambda \in\left(0, \frac{1}{4}\right)$. Since the series $\sum_{n=1}^{\infty} 1 / x_{n}^{1+p}$ converges for all $p>0$ [13] and since $\frac{4}{3}(1-\lambda)>1$, the series $\sum_{n=1}^{\infty} 1 / x_{n}^{4(1-\lambda) / 3}$ converges for all $\lambda<\frac{1}{4}$. Further,

$$
\mathrm{E}\left|\sum_{n=N}^{M} \frac{\sin x_{n} t}{x_{n}} \xi_{n}\right|^{4} \leq\left(\sum_{n=N}^{M} \frac{1}{x_{n}^{4(1-\lambda) / 3}}\right)^{3} \mathrm{E}\left(\sum_{n=N}^{M} \frac{\xi_{n}^{4}}{x_{n}^{4 \lambda}}\right) .
$$

Recall that $\mathrm{E} \xi_{n}^{4}=3\left(\mathrm{E} \xi_{n}^{2}\right)^{2}$, whence we get

$$
\mathrm{E}\left(\sum_{n=N}^{M} \frac{\xi_{n}^{4}}{x_{n}^{4 \lambda}}\right)=3 \sum_{n=N}^{M} \frac{\left(\mathrm{E} \xi_{n}^{2}\right)^{2}}{x_{n}^{4 \lambda}}=3 \sum_{n=N}^{M} \frac{4 c_{H}^{4}}{x_{n}^{4 \lambda+4 H} J_{1-H}^{4}\left(x_{n}\right)} .
$$

Taking into account that $J_{1-H}^{2}\left(x_{n}\right) \sim \frac{2}{\pi x_{n}}$ as $n \rightarrow \infty$ [13], we obtain

$$
\frac{1}{x_{n}^{4 \lambda+4 H} J_{1-H}^{4}\left(x_{n}\right)} \sim \frac{\pi^{2}}{4} \frac{x_{n}^{2}}{x_{n}^{4 \lambda+4 H}}=\frac{\pi^{2}}{4} \frac{1}{x_{n}^{4 \lambda+4 H-2}} .
$$

If $H>\frac{1}{2}$, then there exists a number $\lambda<\frac{1}{4}$ such that $4 \lambda+4 H-2>1$. Now we conclude from $\sum_{n=1}^{\infty} 1 / x_{n}^{1+p}<+\infty$ for all $p>0$ that the series

$$
\sum_{n=1}^{\infty}\left(x_{n}^{4 \lambda+4 H} J_{1-H}^{4}\left(x_{n}\right)\right)^{-1}
$$

converges. Hence

$$
\mathrm{E}\left(\sum_{n=N}^{M} \frac{\sin x_{n} t}{x_{n}} \xi_{n}\right)^{4} \rightarrow 0, \quad N, M \rightarrow \infty
$$

and the series $\sum_{n=1}^{\infty}\left(\sin x_{n} t / x_{n}\right) \xi_{n}$ converges in $L_{4}(\Omega)$ by the Cauchy criterion.

Next we prove that the increments of the first and second order of the series

$$
\sum_{n=1}^{\infty}\left(\sin x_{n} t / x_{n}\right) \xi_{n}
$$

belong to the class $K_{1}$. Let

$$
X(t)=\sum_{n=1}^{\infty} \varphi_{n}(t) \xi_{n}, \quad t \in[0,1],
$$


where $\varphi_{n}(t)=\sin x_{n} t / x_{n}, n \geq 1$. For arbitrary segments $[s, t],[u, v] \subset[0,1]$ without joint inner points put

$$
\begin{gathered}
X_{1, N}=\sum_{n=1}^{N} \rho_{n} \xi_{n}, \quad \text { where } \rho_{n}=\varphi_{n}(t)-\varphi_{n}(s), \\
X_{2, N}=\sum_{n=1}^{N} \tau_{n} \xi_{n}, \quad \text { where } \tau_{n}=\varphi_{n}(v)-\varphi_{n}(u), \quad n \geq 1 .
\end{gathered}
$$

Our aim is to prove that

$$
\mathrm{E}\left(X_{1, N} \pm X_{2, N}\right)^{4}=3\left(\mathrm{E}\left(X_{1, N} \pm X_{2, N}\right)^{2}\right)^{2}
$$

Since $X_{1, N} \pm X_{2, N}=\sum_{n=1}^{N} \mu_{n} \xi_{n}$, where $\mu_{n}=\rho_{n} \pm \tau_{n}, 1 \leq n \leq N$, we get

$$
\begin{aligned}
\mathrm{E}\left(\sum_{n=1}^{N} \mu_{n} \xi_{n}\right)^{4} & =\sum_{n=1}^{N} \mu_{n}^{4} \mathrm{E} \xi_{n}^{4}+6 \sum_{\substack{n, m=1 \\
n<m}}^{N} \mu_{n}^{2} \mu_{m}^{2} \mathrm{E} \xi_{n}^{2} \mathrm{E} \xi_{m}^{2} \\
& =3\left(\sum_{n=1}^{N} \mu_{n}^{4}\left(\mathrm{E} \xi_{n}^{2}\right)^{2}+2 \sum_{\substack{n, m=1 \\
n<m}}^{N} \mu_{n}^{2} \mu_{m}^{2} \mathrm{E} \xi_{n}^{2} \mathrm{E} \xi_{m}^{2}\right) \\
& =3\left(\sum_{n=1}^{N} \mu_{n}^{2} \mathrm{E} \xi_{n}^{2}\right)^{2}=3\left(\mathrm{E}\left(\sum_{n=1}^{N} \mu_{n} \xi_{n}\right)^{2}\right)^{2}
\end{aligned}
$$

Let $X_{i}=\lim _{N \rightarrow \infty} X_{i, N}$ in $L_{4}(\Omega)$ for $i=1,2$. Passing to the limit in (5) as $N \rightarrow \infty$ we conclude that $\mathrm{E}\left(X_{1} \pm X_{2}\right)^{4}=3\left(\mathrm{E}\left(X_{1} \pm X_{2}\right)^{2}\right)^{2}$. Thus $X(t)$ is a stochastic process with increments of the first and second order belonging to the class $K_{1}$. Similarly,

$$
Y(t)=\sum_{n=1}^{\infty}\left(\left(1-\cos y_{n} t\right) / y_{n}\right) \eta_{n}, \quad t \in[0,1]
$$

is a stochastic process with increments of the first order of the class $K_{1}$. Then,

$$
\xi(t)=X(t)+Y(t), \quad t \in[0,1],
$$

is a stochastic process with increments of the first order belonging to the class $K_{1}$. Indeed, for $X_{1}=X(t)-X(s), X_{2}=X(v)-X(u)$ and $Y_{1}=Y(t)-Y(s), Y_{2}=Y(v)-Y(u)$ we have

$$
\begin{aligned}
& \mathrm{E}\left(\left(X_{1}+Y_{1}\right) \pm\left(X_{2}+Y_{2}\right)\right)^{4}=\mathrm{E}\left(\left(X_{1} \pm X_{2}\right)+\left(Y_{1} \pm Y_{2}\right)\right)^{4} \\
& \quad=3\left(\left(\mathrm{E}\left(X_{1} \pm X_{2}\right)^{2}\right)^{2}+2 \mathrm{E}\left(X_{1} \pm X_{2}\right)^{2} \mathrm{E}\left(Y_{1} \pm Y_{2}\right)^{2}+\left(\mathrm{E}\left(Y_{1} \pm Y_{2}\right)^{2}\right)^{2}\right) \\
& \quad=3\left(\mathrm{E}\left(X_{1} \pm X_{2}\right)^{2}+\mathrm{E}\left(Y_{1} \pm Y_{2}\right)^{2}\right)^{2}=3\left(\mathrm{E}\left(\left(X_{1} \pm X_{2}\right)+\left(Y_{1} \pm Y_{2}\right)\right)^{2}\right)^{2} \\
& \quad=3\left(\mathrm{E}\left(\left(X_{1}+Y_{1}\right) \pm\left(X_{2}+Y_{2}\right)\right)^{2}\right)^{2} .
\end{aligned}
$$

We prove similarly that $\xi(t)=X(t)+Y(t), t \in[0,1]$, is a stochastic process with increments of the second order of the class $K_{1}$. 


\section{Strongly COnsistent estimator of the Parameter $H$}

For a stochastic process $\{\xi(t), t \in[0,1]\}$, we introduce the following notation

$$
\begin{gathered}
\xi_{k, n}^{(1)}=\xi\left(\frac{k+1}{a_{n}}\right)-\xi\left(\frac{k}{a_{n}}\right), \\
\xi_{k, n}^{(2)}=\xi\left(\frac{k+1}{a_{n}}\right)-2 \xi\left(\frac{k+0.5}{a_{n}}\right)+\xi\left(\frac{k}{a_{n}}\right), \quad 0 \leq k \leq a_{n}-1 .
\end{gathered}
$$

Consider the sequence of related Baxter sums

$$
S_{n}^{(i)}=\sum_{k=0}^{a_{n}-1}\left(\xi_{k, n}^{(i)}\right)^{2}, \quad \hat{S}_{n}^{(i)}=a_{n}^{2 H-1} S_{n}^{(i)}, \quad i=1,2 .
$$

The following auxiliary result is needed to estimate the variances of the Baxter sums $\hat{S}_{n}^{(i)}, i=1,2$.

Lemma 3.1 (15]). Let a random vector $(\xi, \eta)$ belong to the class $K$. Then

$$
\mathrm{E}\left(\xi^{2} \eta^{2}\right) \leq 2(\mathrm{E} \xi \eta)^{2}+\mathrm{E} \xi^{2} \mathrm{E} \eta^{2}+\frac{1}{2}\left(\left(\mathrm{E} \xi^{2}\right)^{2}-\frac{1}{3} \mathrm{E} \xi^{4}\right)+\frac{1}{2}\left(\left(\mathrm{E} \eta^{2}\right)^{2}-\frac{1}{3} \mathrm{E} \eta^{4}\right)
$$

Lemma 3.1 implies that

$$
\mathrm{E}\left(\xi_{i}^{2} \eta_{i}^{2}\right) \leq 2\left(\mathrm{E}\left(\xi_{i} \eta_{i}\right)\right)^{2}+\mathrm{E} \xi_{i}^{2} \mathrm{E} \eta_{i}^{2}, \quad i=1,2,
$$

where the random vectors $\left(\xi_{1}, \eta_{1}\right)$ and $\left(\xi_{2}, \eta_{2}\right)$ are defined in Definition 2.2 and where a stochastic process $\{\xi(t), t \in[0,1]\}$ has increments of the first and second order belonging to the class $K_{1}$.

Lemma 3.2. Let $H^{*} \in(0,1)$. Then

$$
\sup _{H \in\left(0, H^{*}\right]} \operatorname{Var} \hat{S}_{n}^{(1)} \leq \begin{cases}\frac{2}{a_{n}}\left(3+2 \zeta\left(4-4 H^{*}\right)\right), & \text { if } H^{*} \in\left(0, \frac{3}{4}\right), \\ \left.\frac{2}{a_{n}}\left(3+2\left(1+\ln a_{n}\right)\right)\right), & \text { if } H^{*}=\frac{3}{4}, \\ \frac{2}{a_{n}}\left(3+2 \frac{a_{n}^{4 H^{*}-3}}{4 H^{*}-3}\right), & \text { if } H^{*} \in\left(\frac{3}{4}, 1\right),\end{cases}
$$

where

$$
\zeta(s)=\sum_{n=1}^{\infty} \frac{1}{n^{s}}, \quad s>1 .
$$

Proof. We have

$$
\begin{gathered}
\operatorname{Var} \hat{S}_{n}^{(1)}=a_{n}^{4 H-2} \operatorname{Var} S_{n}^{(1)} \\
\operatorname{Var} S_{n}^{(1)}=\mathrm{E}\left(S_{n}^{(1)}-\mathrm{E} S_{n}^{(1)}\right)^{2}=\mathrm{E}\left(\sum_{k=0}^{a_{n}-1}\left(\xi_{k, n}^{(1)}\right)^{2}-\sum_{k=0}^{a_{n}-1} \mathrm{E}\left(\xi_{k, n}^{(1)}\right)^{2}\right)^{2} \\
=\mathrm{E}\left(\sum_{k, j=0}^{a_{n}-1}\left(\left(\xi_{k, n}^{(1)}\right)^{2}-\mathrm{E}\left(\xi_{k, n}^{(1)}\right)^{2}\right)\left(\left(\xi_{j, n}^{(1)}\right)^{2}-\mathrm{E}\left(\xi_{j, n}^{(1)}\right)^{2}\right)\right) \\
=\sum_{k, j=0}^{a_{n}-1}\left(\mathrm{E}\left(\xi_{k, n}^{(1)}\right)^{2}\left(\xi_{j, n}^{(1)}\right)^{2}-\mathrm{E}\left(\xi_{k, n}^{(1)}\right)^{2} \mathrm{E}\left(\xi_{j, n}^{(1)}\right)^{2}\right) .
\end{gathered}
$$

By inequality (6),

$$
\operatorname{Var} S_{n}^{(1)} \leq 2 \sum_{k, j=0}^{a_{n}-1}\left(\mathrm{E} \xi_{k, n}^{(1)} \xi_{j, n}^{(1)}\right)^{2}
$$


Further,

$$
\mathrm{E} \xi_{k, n}^{(1)} \xi_{j, n}^{(1)}=\frac{1}{2}\left|\frac{(k-j)+1}{a_{n}}\right|^{2 H}-\left|\frac{k-j}{a_{n}}\right|^{2 H}+\frac{1}{2}\left|\frac{(k-j)-1}{a_{n}}\right|^{2 H}, \quad 0 \leq k, j \leq a_{n}-1 .
$$

Put $v_{l}:=(l+1)^{2 H}-2 l^{2 H}+(l-1)^{2 H}, l \geq 1$. Then

$$
\operatorname{Var} S_{n}^{(1)} \leq 2\left(a_{n}^{1-4 H}+\frac{a_{n}^{-4 H}}{2} \sum_{\substack{k, j=0, k<j}}^{a_{n}-1} v_{j-k}^{2}\right) \leq 2 a_{n}^{1-4 H}\left(1+\frac{1}{2} \sum_{l=1}^{a_{n}-1} v_{l}^{2}\right) .
$$

Since $v_{1}^{2}=\left(2^{2 H}-2\right)^{2} \leq 4$ for $H \in(0,1)$, we obtain

$$
\operatorname{Var} S_{n}^{(1)} \leq 2 a_{n}^{1-4 H}\left(3+\frac{1}{2} \sum_{l=2}^{a_{n}-1} v_{l}^{2}\right)
$$

Now $v_{l}$ is an increment of the second order of the function $f(x)=x^{2 H}, x \geq 1$, corresponding to the interval $[l-1, l+1]$. Thus the representation obtained in [9, p. 244] for increments of an arbitrary order implies with $n=2$ that $v_{l}=2 H(2 H-1) \theta_{l}^{2 H-2}$, where $\theta_{l} \in(l-1, l+1)$. Recalling that $\sup _{H \in(0,1)}|2 H(2 H-1)|=2$ we derive

$$
v_{l}^{2} \leq \frac{4}{(l-1)^{4-4 H}}, \quad l \geq 2,
$$

whence

$$
\operatorname{Var} S_{n}^{(1)} \leq 2 a_{n}^{1-4 H}\left(3+2 \sum_{l=2}^{a_{n}-1} \frac{1}{(l-1)^{4-4 H}}\right)
$$

Therefore

$$
\operatorname{Var} \hat{S}_{n}^{(1)} \leq \frac{2}{a_{n}}\left(3+2 \sum_{l=2}^{a_{n}-1} \frac{1}{(l-1)^{4-4 H}}\right)
$$

and

$$
\sup _{H \in\left(0, H^{*}\right]} \operatorname{Var} \hat{S}_{n}^{(1)} \leq \frac{2}{a_{n}}\left(3+2 \sum_{l=2}^{a_{n}-1} \frac{1}{(l-1)^{4-4 H^{*}}}\right)
$$

Since

$$
\sum_{l=1}^{a_{n}} \frac{1}{l^{4-4 H^{*}}} \leq \begin{cases}\zeta\left(4-4 H^{*}\right), & H^{*} \in\left(0, \frac{3}{4}\right) ; \\ 1+\ln a_{n}, & H^{*}=\frac{3}{4} ; \\ \frac{a_{n}^{4 H^{*}-3}}{4 H^{*}-3}, & \text { if } H^{*} \in\left(\frac{3}{4}, 1\right),\end{cases}
$$

where $\zeta(s)=\sum_{n=1}^{\infty} n^{-s}, s>1$, inequality (8) completes the proof of Lemma 3.2

Theorem 3.1. $\hat{S}_{n}^{(1)} \rightarrow 1$ with probability one as $n \rightarrow \infty$.

Proof. Note that

$$
\mathrm{E} \hat{S}_{n}^{(1)}=a_{n}^{2 H-1} a_{n} \mathrm{E}\left(\xi_{0, n}^{(1)}\right)^{2}=1, \quad n \geq 1
$$

In view of the convergence for all $\alpha>0$ of the series $\sum_{n=1}^{\infty} a_{n}^{-\alpha}$, Lemma 3.2 implies that the series $\sum_{n=1}^{\infty} \operatorname{Var} \hat{S}_{n}^{(1)}$ converges for an arbitrary $H \in(0,1)$. Considering (9), we complete the proof of the theorem. 
Lemma 3.3. For an arbitrary $H \in(0,1)$,

$$
\operatorname{Var} \hat{S}_{n}^{(2)} \leq \frac{2}{a_{n}}\left(9+\frac{c}{2}+\frac{c_{*}^{2}}{2} \zeta(4)\right),
$$

where $\zeta(4)=\pi^{4} / 90$,

$$
\begin{gathered}
c=\sup _{H \in(0,1)}\left(\frac{6 \cdot 2^{2 H}-4-4 \cdot 3^{2 H}+2^{4 H}}{2^{2 H}}\right)^{2}, \\
c_{*}=\sup _{H \in(0,1)}\left|\frac{2 H(2 H-1)(2 H-2)(2 H-3)}{2^{4}}\right| .
\end{gathered}
$$

Proof. We have

$$
\operatorname{Var} \hat{S}_{n}^{(2)}=a_{n}^{4 H-2} \operatorname{Var} S_{n}^{(2)} .
$$

As in the proof of Lemma 3.2, we obtain

$$
\operatorname{Var} S_{n}^{(2)} \leq 2 \sum_{k, j=0}^{a_{n}-1}\left(\mathrm{E} \xi_{k, n}^{(2)} \xi_{j, n}^{(2)}\right)^{2}
$$

Further,

$$
\begin{gathered}
\mathrm{E}\left(\xi_{k, n}^{(2)} \xi_{j, n}^{(2)}\right)=\frac{1}{2}\left(-\left|\frac{(k-j)-1}{a_{n}}\right|^{2 H}+4\left|\frac{(k-j)-1 / 2}{a_{n}}\right|^{2 H}-6\left|\frac{k-j}{a_{n}}\right|^{2 H}\right. \\
\left.+4\left|\frac{(k-j)+1 / 2}{a_{n}}\right|^{2 H}-\left|\frac{(k-j)+1}{a_{n}}\right|^{2 H}\right), \\
0 \leq k, j \leq a_{n}-1 .
\end{gathered}
$$

If $k=j$, then

$$
\mathrm{E}\left(\xi_{k, n}^{(2)}\right)^{2}=\mathrm{E}\left(\xi_{0, n}^{(2)}\right)^{2}=a_{n}^{-2 H}\left(2^{2-2 H}-1\right)
$$

Put

$$
w_{l}:=\left(|l-1|^{2 H}-4\left|l-\frac{1}{2}\right|^{2 H}+6 l^{2 H}-4\left|l+\frac{1}{2}\right|^{2 H}+|l+1|^{2 H}\right), \quad l \geq 1 .
$$

If $l=1$, then $w_{1}=\left(6 \cdot 2^{2 H}-4-4 \cdot 3^{2 H}+2^{4 H}\right) /\left(2^{2 H}\right)$. Thus

$$
\begin{aligned}
\operatorname{Var} S_{n}^{(2)} \leq 2 & \left.a_{n}^{1-4 H}\left(2^{2-2 H}-1\right)^{2}+\frac{a_{n}^{-4 H}}{2} \sum_{\substack{k, j=0, k<j}}^{a_{n}-1} w_{j-k}^{2}\right) \\
\leq & 2\left(a_{n}^{1-4 H}\left(2^{2-2 H}-1\right)^{2}\right. \\
& \left.\quad+\frac{a_{n}^{1-4 H}}{2}\left(\frac{6 \cdot 2^{2 H}-4-4 \cdot 3^{2 H}+2^{4 H}}{2^{2 H}}\right)^{2}+\frac{a_{n}^{1-4 H}}{2} \sum_{l=2}^{a_{n}-1} w_{l}^{2}\right) .
\end{aligned}
$$

Since $\sup _{H \in(0,1)}\left(2^{2-2 H}-1\right)^{2}=9$, we conclude that

$$
\operatorname{Var} S_{n}^{(2)} \leq 2 a_{n}^{1-4 H}\left(9+\frac{c}{2}+\frac{1}{2} \sum_{l=2}^{a_{n}-1} w_{l}^{2}\right)
$$


where

$$
c=\sup _{H \in(0,1)}\left(\frac{6 \cdot 2^{2 H}-4-4 \cdot 3^{2 H}+2^{4 H}}{2^{2 H}}\right)^{2} .
$$

Further, $w_{l}$ is an increment of the fourth order of the function $f(x)=x^{2 H}, x \geq 1$, corresponding to the interval $[l-1, l+1]$. Thus the representation for increments of order $n$ with $n=4$ [9, p. 244] implies that

$$
w_{l}=\frac{2 H(2 H-1)(2 H-2)(2 H-3)}{2^{4}} \theta_{l}^{2 H-4}
$$

where $\theta_{l} \in(l-1, l+1)$. Taking into account that

$$
c_{*}=\sup _{H \in(0,1)}\left|\frac{2 H(2 H-1)(2 H-2)(2 H-3)}{2^{4}}\right| \text {, }
$$

we establish

Hence

$$
w_{l}^{2} \leq c_{*}^{2} \frac{1}{(l-1)^{8-4 H}}, \quad l \geq 2 .
$$

$$
\operatorname{Var} S_{n}^{(2)} \leq 2 a_{n}^{1-4 H}\left(9+\frac{c}{2}+\frac{c_{*}^{2}}{2} \sum_{l=2}^{a_{n}-1} \frac{1}{(l-1)^{8-4 H}}\right) \leq 2 a_{n}^{1-4 H}\left(9+\frac{c}{2}+\frac{c_{*}^{2}}{2} \zeta(4)\right),
$$

where

$$
\zeta(4)=\sum_{n=1}^{\infty} n^{-4}=\frac{\pi^{4}}{90}=1.08232323 \ldots
$$

Therefore

$$
\sup _{H \in(0,1)} \operatorname{Var} \hat{S}_{n}^{(2)} \leq \frac{2}{a_{n}}\left(9+\frac{c}{2}+\frac{c_{*}^{2}}{2} \zeta(4)\right) .
$$

Lemma 3.3 is proved.

Theorem 3.2. $\hat{S}_{n}^{(2)} \rightarrow 2^{2-2 H}-1$ with probability one as $n \rightarrow \infty$.

Proof. Note that

$$
\mathrm{E} \hat{S}_{n}^{(2)}=a_{n}^{2 H-1} a_{n} \mathrm{E}\left(\xi_{0, n}^{(2)}\right)^{2}=2^{2-2 H}-1 .
$$

Since the series $\sum_{n=1}^{\infty} a_{n}^{-1}$ converges, Lemma 3.3 implies that the series $\sum_{n=1}^{\infty} \operatorname{Var} \hat{S}_{n}^{(2)}$ converges, too, for an arbitrary $H \in(0,1)$. Theorem 3.2 is proved.

Theorems 3.1 and 3.2 imply the following result.

\section{Corollary 3.1.}

$$
\frac{S_{n}^{(2)}}{S_{n}^{(1)}}=\frac{\hat{S}_{n}^{(2)}}{\hat{S}_{n}^{(1)}} \rightarrow 2^{2-2 H}-1, \quad H \in(0,1),
$$

with probability one as $n \rightarrow \infty$.

Put

$$
\theta(H):=2^{2-2 H}-1, \quad H \in(0,1)
$$

Then

$$
\varphi(\theta)=1-\frac{1}{2} \log _{2}(\theta+1), \quad \theta \in(0,3),
$$

is the inverse function to $\theta(H), H \in(0,1)$. 
Theorem 3.3. The statistic

$$
\hat{H}_{n}=1-\frac{1}{2} \log _{2}\left(\theta_{n}+1\right), \quad n \geq 1,
$$

where $\theta_{n}=S_{n}^{(2)} / S_{n}^{(1)}, n \geq 1$, is a strongly consistent estimator of the parameter $H$.

Proof. Since (14) is the inverse function to (13), convergence (12) implies that $\hat{H}_{n} \rightarrow H$ with probability one as $n \rightarrow \infty$.

\section{Confidence intervals}

Lemma 4.1. Let $\left\{X_{k} \mid 0 \leq k \leq a_{n}, a_{n} \in N\right\}$ and $\left\{Y_{k} \mid 0 \leq k \leq a_{n}, a_{n} \in N\right\}$ be two sequences of centered random variables with finite moments of fourth order. Assume that

$$
\begin{gathered}
\mathrm{E} X_{k}=\mathrm{E} Y_{k}=0, \quad \mathrm{E} X_{k}^{2}=\mathrm{E} X_{0}^{2}, \quad \mathrm{E} Y_{k}^{2}=\mathrm{E} Y_{0}^{2}, \quad 0 \leq k \leq a_{n} ; \\
S_{1}=\sum_{k=0}^{a_{n}} X_{k}^{2}, \quad S_{2}=\sum_{k=0}^{a_{n}} Y_{k}^{2}, \quad \theta=\frac{\mathrm{E} X_{0}^{2}}{\mathrm{E} Y_{0}^{2}} .
\end{gathered}
$$

Then

$$
\mathrm{P}\left\{\left|\frac{S_{1}}{S_{2}}-\theta\right| \geq \varepsilon\right\} \leq \frac{\operatorname{Var} Q_{1}}{\left(\mathrm{E} Q_{1}\right)^{2}}+\frac{\operatorname{Var} Q_{2}}{\left(\mathrm{E} Q_{2}\right)^{2}}
$$

for an arbitrary $\varepsilon>0$, where $Q_{1}=(\theta-\varepsilon) S_{2}-S_{1}$ and $Q_{2}=S_{1}-(\theta+\varepsilon) S_{2}$.

Proof. We have

$$
\begin{aligned}
\mathrm{P}\left\{\left|\frac{S_{1}}{S_{2}}-\theta\right| \geq \varepsilon\right\} & =\mathrm{P}\left\{\left|S_{1}-\theta S_{2}\right| \geq \varepsilon S_{2}\right\} \\
& =\mathrm{P}\left\{S_{1}-\theta S_{2} \leq-\varepsilon S_{2}\right\}+\mathrm{P}\left\{S_{1}-\theta S_{2} \geq \varepsilon S_{2}\right\} \\
& =\mathrm{P}\left\{Q_{1} \geq 0\right\}+\mathrm{P}\left\{Q_{2} \geq 0\right\} .
\end{aligned}
$$

Note that

$$
\begin{aligned}
\mathrm{E} Q_{1} & =(\theta-\varepsilon) \mathrm{E} S_{2}-\mathrm{E} S_{1}=(\theta-\varepsilon)\left(a_{n}+1\right) \mathrm{E} Y_{0}^{2}-\left(a_{n}+1\right) \mathrm{E} X_{0}^{2} \\
& =\left(a_{n}+1\right)(\theta-\varepsilon-\theta)=-\varepsilon\left(a_{n}+1\right) \mathrm{E} Y_{0}^{2}<0
\end{aligned}
$$

The probability $\mathrm{P}\left\{Q_{1} \geq 0\right\}$ is estimated from above by the Chebyshev inequality:

$$
\mathrm{P}\left\{Q_{1} \geq 0\right\}=\mathrm{P}\left\{Q_{1}-\mathrm{E} Q_{1} \geq-\mathrm{E} Q_{1}\right\} \leq \mathrm{P}\left\{\left|Q_{1}-\mathrm{E} Q_{1}\right| \geq-\mathrm{E} Q_{1}\right\} \leq \frac{\operatorname{Var} Q_{1}}{\left(\mathrm{E} Q_{1}\right)^{2}} .
$$

The probability $\mathrm{P}\left\{Q_{2} \geq 0\right\}$ is estimated similarly.

Using Lemma 4.1 we construct the confidence interval for the parameter $\theta=2^{2-2 H}-1$, $H \in(0,1)$. Let $1-p \in(0,1)$ be a given confidence level. A positive number $\gamma_{n}(p)$ is defined as follows

$$
\mathrm{P}\left\{\left|\theta_{n}-\theta\right| \geq \gamma_{n}(p)\right\}<p
$$

Put

$$
Q_{1}=\left(\theta-\gamma_{n}(p)\right) \hat{S}_{n}^{(1)}-\hat{S}_{n}^{(2)}, \quad Q_{2}=\hat{S}_{n}^{(2)}-\left(\theta+\gamma_{n}(p)\right) \hat{S}_{n}^{(1)},
$$

where $\gamma_{n}(p)$ is not specified yet. The expectations of the random variables $Q_{1}$ and $Q_{2}$ are such that

$$
\mathrm{E} Q_{1}=\mathrm{E} Q_{2}=-\gamma_{n}(p)
$$


We use the inequality $(a+b)^{2} \leq 2\left(a^{2}+b^{2}\right), a, b \in \mathbf{R}$, to estimate the variances of the random variables $Q_{1}$ and $Q_{2}$ :

$$
\begin{aligned}
& \operatorname{Var} Q_{1} \leq 2\left(\theta-\gamma_{n}(p)\right)^{2} \operatorname{Var} \hat{S}_{n}^{(1)}+2 \operatorname{Var} \hat{S}_{n}^{(2)}, \\
& \operatorname{Var} Q_{2} \leq 2\left(\theta+\gamma_{n}(p)\right)^{2} \operatorname{Var} \hat{S}_{n}^{(1)}+2 \operatorname{Var} \hat{S}_{n}^{(2)} .
\end{aligned}
$$

The estimates for $\operatorname{Var} \hat{S}_{n}^{(1)}$ and $\operatorname{Var} \hat{S}_{n}^{(2)}$ are proved in Lemmas 3.2 and 3.3. respectively. Recall that $\theta \in(0,3)$.

We choose $\gamma_{n}(p)$ such that

$$
\begin{aligned}
& \frac{\operatorname{Var} Q_{1}}{\left(\mathrm{E} Q_{1}\right)^{2}} \leq \frac{2\left(\theta-\gamma_{n}(p)\right)^{2} \operatorname{Var} \hat{S}_{n}^{(1)}+2 \operatorname{Var} \hat{S}_{n}^{(2)}}{\gamma_{n}^{2}(p)} \leq \frac{p}{2}, \\
& \frac{\operatorname{Var} Q_{2}}{\left(\mathrm{E} Q_{2}\right)^{2}} \leq \frac{2\left(\theta+\gamma_{n}(p)\right)^{2} \operatorname{Var} \hat{S}_{n}^{(1)}+2 \operatorname{Var} \hat{S}_{n}^{(2)}}{\gamma_{n}^{2}(p)} \leq \frac{p}{2}
\end{aligned}
$$

For $H^{*} \in(0,1)$, put

$$
\begin{gathered}
\alpha_{n}= \begin{cases}\frac{4}{a_{n}}\left(3+2 \zeta\left(4-4 H^{*}\right)\right), & H^{*} \in\left(0, \frac{3}{4}\right) ; \\
\left.\frac{4}{a_{n}}\left(3+2\left(1+\ln a_{n}\right)\right)\right), & H^{*}=\frac{3}{4} ; \\
\frac{4}{a_{n}}\left(3+2 \frac{a_{n}^{4 H^{*}-3}}{4 H^{*}-3}\right), & H^{*} \in\left(\frac{3}{4}, 1\right),\end{cases} \\
\beta_{n}=\frac{4}{a_{n}}\left(9+\frac{c}{2}+\frac{c_{*}}{2} \zeta(4)\right),
\end{gathered}
$$

where $c, c_{*}, \zeta(s), s>1$, are defined by equalities (10), (11), and (7), respectively.

Now Lemmas 3.2 and 3.3 imply that

$$
\alpha_{n} \leq \sup _{H \in\left(0, H^{*}\right]} \operatorname{Var} \hat{S}_{n}^{(1)}, \quad \beta_{n} \leq \sup _{H \in\left(0, H^{*}\right]} \operatorname{Var} \hat{S}_{n}^{(2)} .
$$

If $H \in\left(0, H^{*}\right]$, then inequality (16) follows from the inequality

$$
\frac{\left(3+\gamma_{n}(p)\right)^{2} \alpha_{n}+\beta_{n}}{\gamma_{n}^{2}(p)} \leq \frac{p}{4} .
$$

Solving this inequality with respect to $\gamma_{n}(p)$ under the condition that $\frac{p}{4}-\alpha_{n}>0$ (which is true for all $n$ sufficiently large) we get

$$
\gamma_{n}(p) \geq \frac{6 \alpha_{n}+\sqrt{D_{n}}}{2\left(\frac{p}{4}-\alpha_{n}\right)}
$$

where

$$
D_{n}=36 \alpha_{n}^{2}+4\left(\frac{p}{4}-\alpha_{n}\right)\left(9 \alpha_{n}+\beta_{n}\right)
$$

Put

$$
\gamma_{n}(p)=\frac{6 \alpha_{n}+\sqrt{D_{n}}}{2\left(\frac{p}{4}-\alpha_{n}\right)} .
$$

The above reasoning implies the following result.

Theorem 4.1. Let $H \in\left(0, H^{*}\right]$, where $H^{*} \in(0,1)$ is a fixed number, and let

$$
\frac{p}{4}-\alpha_{n}>0 \text {. }
$$

Then $\left(H_{n, l}, H_{n, r}\right)$ is a confidence interval for the parameter $H$ with the confidence probability $1-p \in(0,1)$, where

$$
H_{n, l}=\varphi\left(\min \left(\theta_{n}+\gamma_{n}(p), 3\right)\right), \quad H_{n, r}=\varphi\left(\max \left(\theta\left(H^{*}\right), \theta_{n}-\gamma_{n}(p)\right)\right),
$$


$\theta_{n}=S_{n}^{(2)} / S_{n}^{(1)}$, the function $\varphi(H)$ is defined by equality (14), and $\gamma_{n}(p)$ is evaluated by (17).

Note that $\gamma_{n}(p)=O\left(\sqrt{\alpha_{n}}\right)$ as $n \rightarrow \infty$.

\section{Concluding Remarks}

A Baxter type estimator of the unknown parameter $H$ of the covariance function (10) of a stochastic process whose first and second order increments belong to the class $K_{1}$ is studied in this paper. Non-asymptotic confidence intervals for $H$ are also found.

\section{BIBLIOGRAPHY}

1. E. P. Besklinskaya and Yu. V. Kozachenko, Convergence in normed Orlicz spaces and LévyBaxter theorems, Teor. Imovir. Mat. Stat. 35 (1986), 3-6; English transl. in Theory Probab. Math. Statist. 35, (1987), 1-5. MR885658 (88f:60076)

2. V. V. Buldygin and Yu. V. Kozachenko, Metric Characterization of Random Variables and Random Processes, TViMS, Kiev, 1998; English transl., American Mathematical Society, Providence, R. I., 2000. MR:1743716 (2001g:60089)

3. E. G. Gladyshev, A new limit theorem for stochastic processes with Gaussian increments, Teor. Veroyatnost. i Primenen. 6 (1961), 57-66; English transl. in Theory Probab. Appl. 6 (1960), no. 1, 52-61. MR0145574(26:3104)

4. Yu. V. Kozachenko and O. O. Kurchenko, Estimation of the parameters of Gaussian homogeneous random fields, Ukrain. Math. Zh. 52 (2000), no. 8, 1082-1088; English transl. in Ukrain. Math. J. 52 (2000), no. 8, 1239-1246. MR1819718(2002a:62139)

5. S. M. Krasnitskiu, Certain limit theorems for random fields with Gaussian differences of order $m$, Teor. Veroyatnost. Mat. Stat. 5 (1971), 71-80; English transl. in Theory Probab. Math. Statist. 5 (1971), 73-82. MR0287604 (44:4807)

6. O. O. Kurchenko, A strongly consistent estimate for the Hurst parameter of fractional Brownian motion, Teor. Imovir. Mat. Stat. 67 (2002), 45-54; English transl. in Theory Probab. Math. Statist. 67 (2003), 88-96. MR.1956622(2005f:62043)

7. O. O. Kurchenko, A Baxter type theorem for strongly sub-Gaussian random fields, Visnyk Kyiv Nats. Taras Shevchenko Univer. Ser. Matem. Mekh. 5 (2009), 35-38. (Ukrainian)

8. R. E. Maiboroda, The asymptotic normality of Baxter estimates for the parameters of nonstationary processes, Teor. Imovir. Mat. Stat. 53 (1995), 97-102; English transl. in Theory Probab. Math. Statist. 53 (1996), 107-112. MR1449110(98d:62148)

9. G. M. Fikhtengol'ts, A Course of Differential and Integral Calculus, vol. 1, "Nauka", Moscow, 1969. (Russian)

10. G. Baxter, A strong limit theorem for Gaussian processes, Proc. Amer. Math. Soc. 7 (1956), no. 3, 522-527. MR0090920(19:890f)

11. S. M. Berman, A version of the Lévy-Baxter theorem for the increments of Brownian motion of several parameters, Proc. Amer. Math. Soc. 18 (1967), 1051-1055. MR0222958 (36:6008)

12. J-C. Breton, I. Nourdin, and G. Peccati, Exact confidence intervals for the Hurst parameter of a fractional Brownian motion, Electronic J. Statist. 3 (2009), 416-425. MR2501319(2010f:62074)

13. K. Dzhaparidze and J. H. van Zanten, A series expansion of fractional Brownian motion, Probab. Theory Related Fields 130 (2004), no. 1, 39-55. MR2092872 (2005i:60065)

14. T. Kawada, The Lévy-Baxter theorem for Gaussian random fields: A sufficient condition, Proc. Amer. Math. Soc. 53 (1975), 463-469. MR0383512 (52:4393)

15. Y. V. Kozachenko and O. O. Kurchenko, Lévy-Baxter theorems for one class of non-Gaussian stochastic processes, Random Oper. Stoch. Equ. 4 (2011), 313-326. MR.2871844

16. P. Lévy, Le mouvement Brownian plan, Amer J. Math. 62 (1940), 487-550. MR0002734 $(2: 107 \mathrm{~g})$

Department of Probability Theory, Statistics, and Actuarial Mathematics, Faculty for Mechanics and Mathematics, National Taras Shevchenko University, Volodymyrs'ka Street, 64, KYIV 01601, UKRAINE

E-mail address: olja_sunjavska@ua.fm 